\title{
Development and repair of experimental pressure ulcers in the rat abdominal wall induced by repeated compression using magnets*
}

\author{
Seiichi Kawamata, Tomoyuki Kurose, Yuta Honkawa, Yohei Kubori, and \\ Hiroaki Muramoto \\ Department of Anatomy and Histology, Institute of Biomedical \& Health Sciences, Hiroshima University, Hiroshima, \\ Japan
}

\begin{abstract}
Summary. A better understanding of pressure ulcers requires animal models under precise experimental conditions. We here report on an improved and clinically more relevant model of pressure ulcers. Rats underwent implantation of a gold-plated magnet $(25 \times 20 \times 2 \mathrm{~mm})$ into their peritoneal cavities under anesthesia. At either 3 or 4 days postoperatively, the rat abdominal wall was repeatedly compressed at $100 \mathrm{mmHg}(13.3 \mathrm{kPa})$ for $4 \mathrm{~h}$ daily for 5 consecutive days by applying another magnet to the skin while animals were conscious. The rats were reared without further treatment and the abdominal skin was photographed daily. Specimens were removed at appropriate intervals for histological examination. Edema and mild redness of the skin appeared at 1 day after the first compression. After a few repetitions of compression, edema and redness of the skin were enhanced. Subsequently, in the majority of rats, redness
\end{abstract}

Received January, 20, 2012

*This study was supported by Grants-in-Aid from the Japan Society for the Promotion of Science (Nos.19592492, and 22500461 to S.K.; Nos. 19700435, 21700533 and 23700602 to T.K.).

Address for correspondence: Seiichi Kawamata, M.D., Ph.D., Department of Anatomy and Histology, Institute of Biomedical \& Health Sciences, Hiroshima University, Kasumi 1-2-3, Minami-ku, Hiroshima 734-8551, Japan

Tel: +81-82-257-5410, Fax: +81-82-257-5410

E-mail: kawamat@hiroshima-u.ac.jp of the skin proceeded to full-thickness necrosis and/or discoloration throughout or in part of the compressed area. Necrotic skin was replaced by black/brown eschar, accompanying exudation, and significant tissue hardening. The injuries considerably contracted a few days after completing the series of compressions. The eschar further decreased gradually in size and usually became detached with time, after which a skin ulcer formed. A small number of rats showed only erosion and/or severe redness of the skin, damage which was reversible to heal without eschar formation. Generally, skin injuries seemed most severe a few days after 5 compressions and essentially healed over the subsequent 20 days. This model should prove useful for developing preventive measures and treating pressure ulcers.

\section{Introduction}

Pressure ulcers constitute a major problem for persons who are unable or find it difficult to change the position of their bodies. Studying the pathogenesis and repair process of pressure ulcers is of great clinical importance to prevent and treat pressure ulcers. However, the mechanisms of development and progression of pressure ulcers are not fully understood because numerous factors are involved in their production (Stekelenburg et al., 2005), including the magnitude of pressure, exposure time, friction, shear forces (Linder-Ganz and Gefen, 2004, 2007), and temperature (Kokate et al., 1995). Furthermore, the condition of patients varies considerably in terms of age, body weight, nutrition (Cordeiro et al., 2005), 
medical history, chronic disease and medication. Therefore, a suitable animal model of pressure ulcers is necessary to examine the development and repair process of pressure ulcers under controlled, and precise experimental conditions.

In early experiments, heavy weights and pressure applicators (e.g., indenters) were used (Kosiak, 1959; Daniel et al., 1981; Bosboom et al., 2001; Linder-Ganz and Gefen, 2004; Stekelenburg et al., 2006). These methods required the animals to be anesthetized during experiments to immobilize them. In addition, clinically irrelevant magnitudes and/or times of exposure to pressure were often used (Daniel et al., 1981; Bosboom et al., 2001; Lin et al., 2010). However, anesthesia may compromise the true effects of pressure (Peirce et al., 2000; Stekelenburg et al., 2005). Hashimoto et al. (2008) clearly demonstrated that pentobarbital anesthesia significantly alleviated pressure-induced injuries and stated that experimental pressure ulcers should be examined under waking conditions to avoid effects of anesthetics.

In experiments in a waking condition, magnets have been used for compression. Peirce et al. (2000), Reid et al. (2004) and Wassermann et al. (2009) used magnets for models of pressure ulcers. They implanted a magnet or a steel plate into the subcutaneous connective tissue of the back or under a muscle (Wassermann et al., 2009) and caused compression by applying a magnet to the skin. Saito $e t$ al. (2008) pulled up mouse skin, pinched it using two magnets for $12 \mathrm{~h} 3$ times and observed the repair process of pressure ulcers. In our previous study (Hashimoto et al., 2008), the rat abdominal wall was compressed at $100 \mathrm{mmHg}(13.3 \mathrm{kPa})$ for $4 \mathrm{~h}$ using magnets; this demonstrated that magnet compression is an appropriate model for studying the pathogenesis of pressure ulcers. Unfortunately, in this study, compression was possible only one time for the reason mentioned below. The result was that edema and redness of the skin were produced, but not skin necrosis or breakdown. Next, we attempted repeated compression. However, when a magnet was implanted for more than 1 day into the peritoneal cavity, gastrointestinal problems occurred, presumably due to the nickel that covers the surface of neodymium magnets. Thus, gold-plated neodymium magnets were used for implantation in the present study, and skin necrosis and breakdown were successfully produced by repeated compression. Using this model, we report on the degeneration and repair processes.

\section{Materials and Methods}

A total of 32 Wistar male rats $(265 \pm 12 \mathrm{~g}$, mean \pm standard deviation) aged 8 and 9 weeks were subjected to a series of 5 compressions. Additionally, 3 agematched Wistar males were used in an untreated condition, 1 day after 2 compressions and 1 day after 4 compressions, to compare the microscopic findings of the abdominal wall. The present experimental procedures were approved by the Committee of Research Facilities for Laboratory Animal Science, Hiroshima University. The present study used the method reported by Hashimoto et al. (2008) with a minor modification in that gold-plated magnets were implanted instead of nickel-plated magnets. In short, the rats were anesthetized with ether and hairs were cut with an electric clipper. After disinfection of the skin, a right-to-left incision (about $20 \mathrm{~mm}$ ) was made in the lower abdomen at 60 $\mathrm{mm}$ caudal from the xiphoid process and a thin sterilized gold-plated magnet $(25 \times 20 \times 2 \mathrm{~mm}$, approximately $7.5 \mathrm{~g}$, NeoMag, Ichikawa) was implanted into the peritoneal cavity. Later in the experiment, premagnetized magnet was gold-plated with a tougher gold layer by another company (Sanyo Mekki Kogyo, Hiroshima) and magnetized by the magnet company (NeoMag) because electroplating of magnets is technically difficult. The gold layer of the magnets was critically important since, if there were any defects in the gold layer, neodymium magnets would easily rust, resulting in tissue adhesion, gastrointestinal problems, and emaciation of rats leading to death. After suturing the abdominal incision, the animals were returned to their cages and reared individually to avoid cross-reactivity of magnets, with free access to water and food.

After either 3 or 4 days postoperatively, the rats were anesthetized with ether, marked with a felt pen on the area of the abdominal skin to be compressed (i.e., a rectangle of $25 \mathrm{~mm}$ in length $\times 20 \mathrm{~mm}$ in width, $5 \mathrm{~mm}$ caudal to the xiphoid process; Hashimoto et al., $2008)$ and a strong neodymium magnet $(25 \times 20 \times 5$ $\mathrm{mm}$, approximately $18.8 \mathrm{~g}$, NeoMag) was applied on the skin. The rat abdominal wall was sandwiched by these two magnets at $100 \mathrm{mmHg}$. The magnitude of pressure was selected to simulate physiological compression stress. The condition of compression was determined on the basis of our previous study (Hashimoto et al., 2008) as well as preliminary experimental data showing the majority of rats did not proceed to skin breakdown after 4 compressions at 100 $\mathrm{mmHg}$ for $4 \mathrm{~h}$. The pressure was precisely adjusted by inserting an acrylic plate(s) of appropriate thickness 
between the rat skin and the strong magnet. For pressure adjustment, we used: 1) two weights (water-containing bottles weighing 680 and $687 \mathrm{~g}$ ); 2) a screw cap of a bottle secured with a strong magnet on top of it using adhesive tape; and 3$)$ a series of acrylic plates $(25 \times 20$ $\mathrm{mm}$ ) with thicknesses differing in increments of $0.1 \mathrm{~mm}$. The weights 680 and $687 \mathrm{~g}$ were equivalent to 100 and $101 \mathrm{mmHg}$ for the compressed area $\left(5 \mathrm{~cm}^{2}\right)$, respectively.

Rats were anesthetized using ether and the compressed area was marked with a felt pen. The rat was laid on a table in a prone position. Forelimbs and hindlimbs of the rat were elevated to expose the abdomen. First, the cap of the bottle weighing $680 \mathrm{~g}$ was screwed on. An acrylic plate(s) was placed on the cap of the weight on the table. Then the rat abdomen was approached with the acrylic plate(s) and strong magnet. The thickness of the inserted acrylic plate(s) was decreased when the weight was not lifted up into the air by the attraction of the intraperitoneal magnets just until the point where the weight was lifted up. Second, the bottle was unscrewed and changed from the 680 to the $687 \mathrm{~g}$ bottle and its cap was screwed on. Then the thickness of the acrylic plate(s) was increased until the weight of the $687 \mathrm{~g}$ bottle was not lifted up by the attraction of the two magnets. After this procedure, the weight was changed again from the 687 to the $680 \mathrm{~g}$ bottle and the thickness of the acrylic plate(s) was adjusted on the order of $0.1 \mathrm{~mm}$. These maneuvers were repeated until the same acrylic plate(s) satisfied two conditions, that is, lifting up a $680 \mathrm{~g}$ weight but not a $687 \mathrm{~g}$ weight (Table 1). Next, the strong magnet was separated from the screw cap and the magnet alone was applied on the acrylic plate(s) placed at a precise area of the abdominal wall with the rat in a supine position for compression. It was then manually ensured that two magnets correctly faced each other. Under this condition, the pressure on the abdominal wall was considered to range between 100 and $101 \mathrm{mmHg}$. Due to their attractive force to each other, magnets never changed their positions during compression. The thickness of the acrylic plate(s) was $5.86 \pm 0.17 \mathrm{~mm}$ at the first compression. Such a thick acrylic plate(s) was employed to minimize the change in pressure during compression due to a thinning of the abdominal wall by pressure. The rats immediately recovered from ether anesthesia, behaved normally, and were subjected to compression at $100 \mathrm{mmHg}$ for $4 \mathrm{~h}$ in waking conditions daily for 5 consecutive days. The $4 \mathrm{~h}$ exposure duration was based on a previous study (Hashimoto et al., 2008). The thickness of the acrylic plate(s) was adjusted daily at the start of compression under ether anesthesia. After 5 compressions, rats were allowed to survive without further treatment with free access to water and food until sampling.

Photographs of the rat abdominal walls were taken daily using a digital camera. Anesthesia was unnecessary to take these photographs.

The degree of injury was divided into three classes: 1) complete or almost complete necrosis (skin necrosis and eschar formation roughly $3 / 4$ or more of the compressed area); 2) partial necrosis (skin necrosis and eschar formation roughly less than 3/4 of the compressed area); and 3) superficial injury (edema, redness and erosion but no eschar formation). When rats were subjected to compression at $50 \mathrm{mmHg}$ for $4 \mathrm{~h}$ daily for 5 consecutive days in another experiment, the majority of the animals showed little skin changes except for some hair loss. This finding indicates that injuries here are attributable to pressure but not to any contact of the magnet on the skin.

\section{Preparation of specimens}

The rats were euthanized using ether for sampling at 1 day after either 2 or 4 compressions, and 2, 4, 8, 12, 16

Table 1. Adjustment of the pressure for compression

\begin{tabular}{lcccc}
\hline & \multicolumn{2}{c}{ Lifting up or not of a weight by the intraperitoneal magnet } \\
\hline Weight & $680 \mathrm{~g}(100 \mathrm{mmHg})$ & no & yes & yes \\
\cline { 2 - 5 } $687 \mathrm{~g}(101 \mathrm{mmHg})$ & no & $100 \leq$ pressure $<101$ & yes \\
\hline Estimated pressure $(\mathrm{mmHg})^{*}$ & pressure $<100$ & appropriate & too thin \\
\hline Acrylic plate(s) & too thick & $\begin{array}{l}\text { separate the magnet } \\
\text { from the cap of } \\
\text { change to } \\
\text { thinner acrylic } \\
\text { pction to be taken }\end{array}$ & $\begin{array}{l}\text { weight and proceed to } \\
\text { compression }\end{array}$ & $\begin{array}{l}\text { change to thicker } \\
\text { acrylic plate(s) }\end{array}$ \\
\hline
\end{tabular}

$*:$ pressure $=$ the attractive force of magnets $/$ the compressed area $\left(5 \mathrm{~cm}^{2}\right)$ 
and 20 days after 5 compressions $(6,8,12,16,20$ and 24 days after the first compression, respectively). Tissue specimens from the compressed area were removed along with the surrounding zones of the uncompressed abdominal wall. The specimen was transversely halved. For precise transverse sectioning, specimens were placed on balsa wood (1 mm thick) which was prefixed using glue at a right angle on a cork disk (about $20 \mathrm{~mm}$ in diameter and several $\mathrm{mm}$ in thickness) and pre-coated with $6 \%$ tragacanth gum jelly. The specimen and balsa wood were additionally supported with tragacanth gum jelly and rapidly frozen together in isopentane cooled with liquid nitrogen. Transverse sections $(10 \mu \mathrm{m}$ in thickness) were cut, stained with hematoxylin and eosin, and observed with an Olympus B51 light microscope (Olympus, Tokyo).

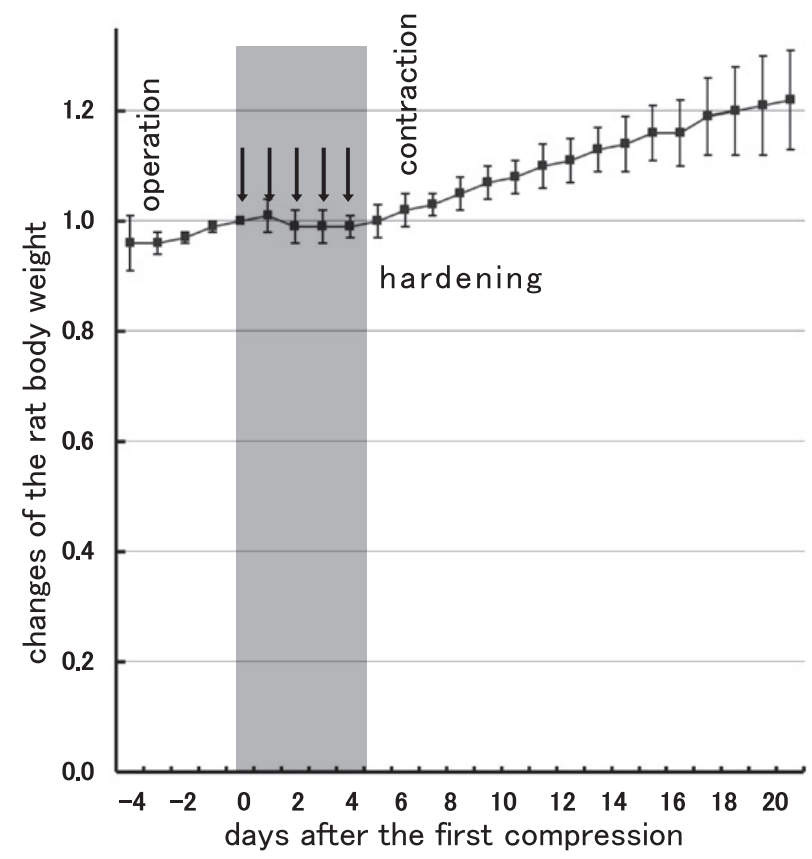

Fig. 1. Relative changes of rat body weights and the time course of the experiment. Body weights decreased slightly during the 5 compression times (shadowed area and arrows) but steadily increased after the operation and after the compression. The body weight of each rat is expressed relative to the body weight at the first compression; the body weight at the first compression has a value of 1 . As rats were sacrificed over time, the standard deviation became larger owing to a decreasing number of rats. "contraction" and "hardening" indicate the periods when contraction and hardening of injured skin were observed, respectively.

\section{Results}

\section{General effects of magnet implantation}

Rats implanted with a gold-plated magnet behaved normally in their cages and continued to develop normally for months as long as the gold layer of the magnet was intact. The body weight of rats was unchanged or slightly decreased during the 5 daily compression times, but this steadily increased after the operation for magnet implantation and after the last compression (Fig. 1). Abdominal organs seemed macroscopically normal when specimens were sampled and gold-plated magnets could move freely in the rat peritoneal cavities. Three out of 32 implanted magnets showed minute rusting or slight elevation of the gold layer, but there were no abnormal findings in the abdominal organs. However, some adhesion of tissue, in particular the inner surface of the compressed abdominal wall and the greater omentum (6 out of 32 rats), was observed when injuries due to compression were severe. When implanted magnets exhibited more apparent changes at specimen sampling, these rats were excluded from the present study.

\section{Macroscopic changes in the abdominal skin after repeated compression}

At 1 day after the first compression, considerable edema and mild redness of the skin appeared. After a few repetitions of compression, edema and redness of the skin were enhanced (Fig. 2). Necrosis appeared, in early cases, at 1 day after 2 or 3 compressions regardless of the following progress of injury. Except for some mild cases, yellowish superficial necrosis at the compressed area proceeded to full-thickness skin necrosis and/or dark discoloration with exudation, followed by black/ brown eschar formation. In 14 out of 32 rats, necrosis and eschar formation was exhibited almost throughout the compressed area (complete or almost complete necrosis, Fig. 2). Twelve out of 32 rats showed necrosis and partial eschar formation to varying degrees in the compressed area (partial necrosis, Fig. 3a, b, c, i). The remaining 6 rats showed only severe redness or superficial necrosis and erosion, which healed without full-thickness skin necrosis (superficial injury, Fig. 3d-h). Generally, skin injuries seemed to be most severe a few days after the 5 compressions.

\section{Hardening of the injured skin}

The abdominal skin became increasingly hard by repeated 


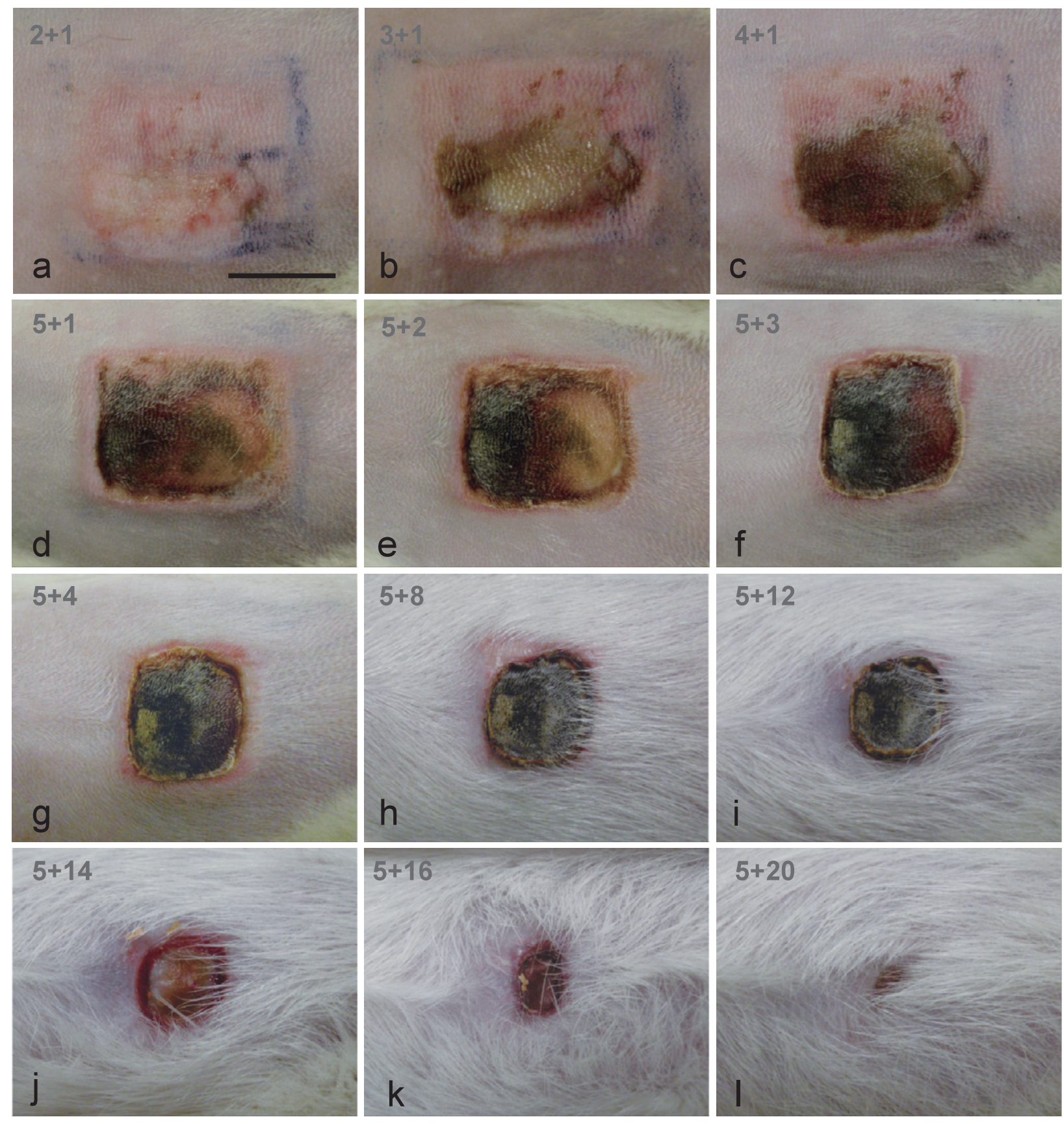

Fig. 2. Sequential photographs of the rat abdominal wall demonstrating complete skin necrosis and eschar formation. The right side of all photographs indicates the rostral direction. The figures at the upper left corner indicate the times of compression and the days after the last compression (e.g., $2+1$ means 1 day after 2 compressions). d e, f : The injured skin apparently contracted from 1 to 3 days after 5 compressions. j: The eschar was detached and a skin ulcer formed. 1: The ulcer has almost healed. Bar $=1 \mathrm{~cm}$ 

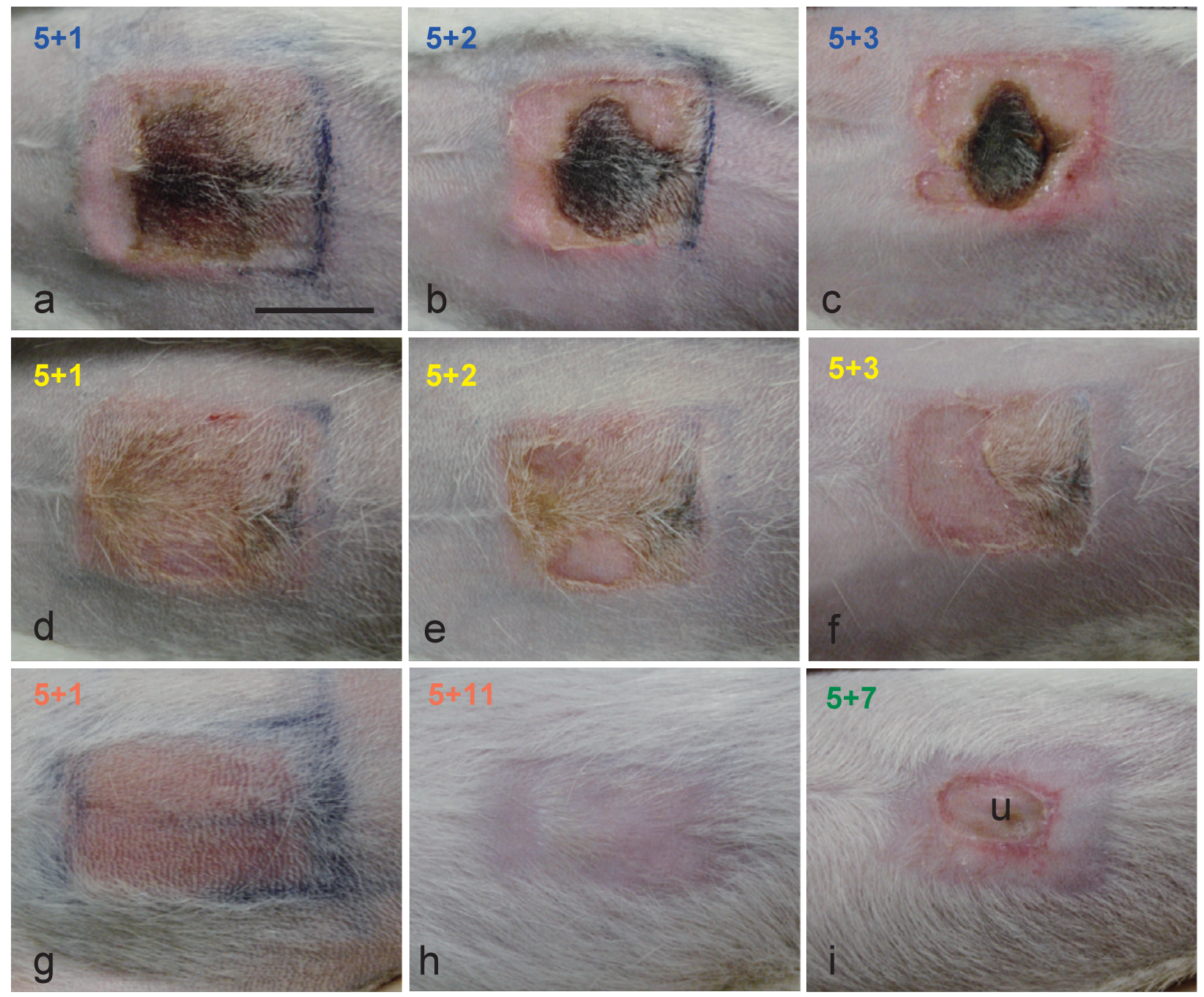

Fig. 3. Photographs showing partial necrosis $(\mathbf{a}, \mathbf{b}, \mathbf{c}, \mathbf{i})$ and superficial injuries $(\mathbf{d}, \mathbf{e}, \mathbf{f}, \mathbf{g}, \mathbf{h})$. The figures at the upper left corner indicate the times of compression and the days after the last compression. The same color of the figures at the upper left corner indicates the same animal. Bar $=1 \mathrm{~cm}$. g: Redness is apparent, but no necrosis is observable. h: Hair loss is found at the compressed area at 10 days after $\mathbf{g}$. i: Hair loss is seen around an ulcer (u)

compression at various times after onset, as early as after a few compressions. The abdominal skin of most rats reached a very hard state like wooden board at 1 day after the 5 compressions and usually remained very hard for about 1 week (Fig. 1).

\section{Contraction of the injured skin}

The compressed area slightly changed in size and shape during the series of 5 compressions while the dark- colored injured area was apparently reduced in size (contraction) from 1 to 3 days after the 5 compressions. The decrease in length was greater in a rostral-caudal direction than that of the width. The shape of the injured area then changed from rectangular to square (Fig. 2, 3), and thereafter gradually to round. With time, the hard black eschar was steadily reduced in size and usually detached at 4 days or later after the 5 compressions with considerable differences among rats, leaving a deep ulcer extending from the skin through to the abdominal 
muscle. No perforation of the abdominal wall was experienced. The ulcer slowly diminished. At 20 days after 5 compressions ( 24 days after the first compression), 3 out of 7 rats that had suffered complete or partial necrosis significantly healed, leaving a small ulcer of less than several $\mathrm{mm}$ in diameter (Fig. 2). The injuries of the remaining 4 rats were covered with epithelium and healed. Varying degrees of hair loss were noticed at 1 week or later after 5 compressions, particularly in rats with mild and moderate skin injuries (i.e., swelling, redness, erosion and partial eschar formation) (Fig. 3).

\section{Changes in thickness of the inserted acrylic plate(s)}

The thicknesses of the acrylic plate(s) between the rat skin and the strong magnet at the start of 100 $\mathrm{mmHg}$ compression were $5.86 \pm 0.17 \mathrm{~mm}$ at the first compression, $4.83 \pm 0.52 \mathrm{~mm}$ at the second compression, $4.10 \pm 0.35 \mathrm{~mm}$ at the third compression, $4.10 \pm 0.43$ $\mathrm{mm}$ at the fourth compression, and $3.96 \pm 0.46 \mathrm{~mm}$ at the fifth compression $(n=27$, each). The time course of the thickness of the acrylic plate(s) is summarized in Figuer 4.

\section{Microscopic findings}

The abdominal walls of untreated normal rats were composed of the following layers: 1) the skin consisting of the epidermis, dermis and skin muscle (panniculus carnosus, often lacking near the midline); 2) the subcutaneous connective tissue; 3 ) the epimysium and abdominal muscles; and 4) the peritoneum (Fig. 5a). Numerous hair follicles and sebaceous glands were observed in the dermis. Adipocytes were seen in the dermis and subcutaneous connective tissue.

At 1 day after 2 compressions, the dermis and subcutaneous connective tissue were very thick, edematous and stained less intensely. Hair follicles had almost disappeared, in agreement findings by Şener et al. (2006) and Saito et al. (2008). Sebaceous glands were damaged and small in size. Skin muscles were fragmented or had almost disappeared. In the subcutaneous connective tissue, cells were scarce and eosinophilic fibrillar material ran generally parallel to the skin. In the abdominal muscle, interstitial space was increased among degenerated or rounder muscle fibers. Myonuclei were invisible or faint. Infiltrated cells in the degenerated muscle fibers were rare.

At 1 day after 4 compressions, the dermis and subcutaneous connective tissue were very thick and edematous. Although there were few damaged sebaceous glands, general findings were similar to those at 1 day after 2 compressions. The epidermis was detached from the dermis more often. In some cases, necrotic epidermis and dermis were found above the eosinophilic fibrillar material. Muscle fibers were broken down into small pieces; however, infiltrated cells were sparse among them.

At 2 days after 5 compressions, the general findings were similar to those at 1 day after 4 compressions. In some cases, however, small regenerating muscle fibers were observed in the abdominal muscle, and almost normal dermis and skin muscle covered degenerated subcutaneous connective tissue at the border of compressed and non-compressed areas.

At 4 days after 5 compressions, although the findings of the skin were in general agreement with those at 2 days after 5 compressions, some regeneration was observed. Cell density was increased in the subcutaneous connective tissue. The skin muscle was regenerated in the vicinity of the normal skin area. Muscle fibers in the abdominal muscle were also regenerating. In a few cases, a mass of necrotic muscles was surrounded by a zone of very densely packed infiltrated cells, probably leucocytes (not

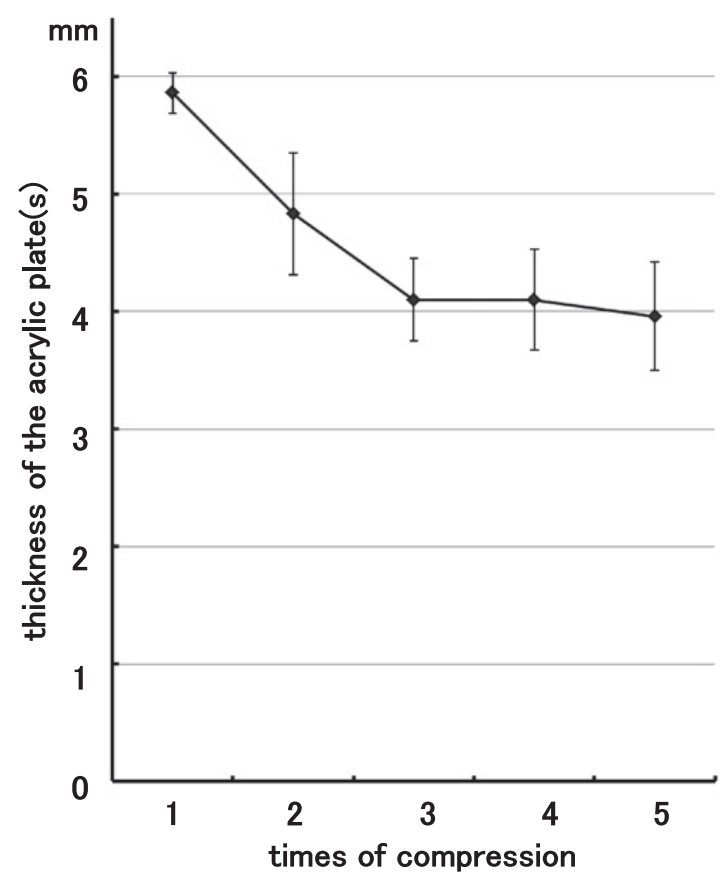

Fig. 4. Changes in the thickness (means \pm standard deviation, $n=27$ ) of acrylic plate(s) between the rat skin and a strong magnet. The thickness was markedly decreased after the first and second compressions but essentially unchanged thereafter. 


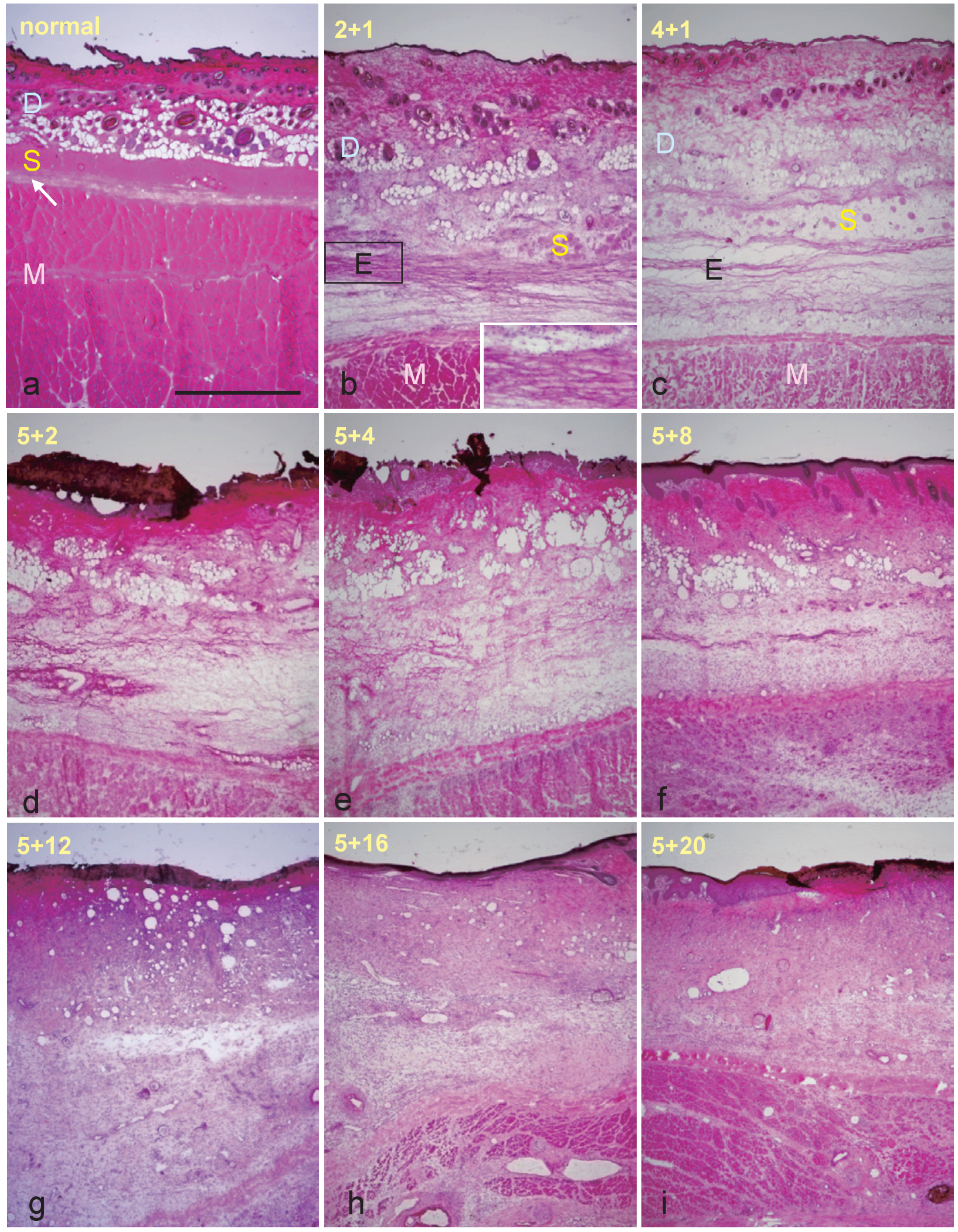


shown).

At 8 days after 5 compressions, the surface of wounds was often covered with epithelium. However, there were few hair follicles and few small sebaceous glands. In the subcutaneous connective tissue, there was only a little eosinophilic fibrillar material and cells were increased in density. In the abdominal muscle, degenerated and regenerated muscle fibers coexisted.

At 12 days after 5 compressions, the epidermis was often regenerated, but the hair follicles and sebaceous glands were still scarce. Regenerating small muscle fibers were observed with considerable differences among rats.

At 16 and 20 days after 5 compressions, the epidermis was often regenerated. However, normal structure of the dermis and subcutaneous connective tissue was not restored.

\section{Discussion}

In the present study, various skin injuries such as edema, redness, erosion, discoloration, skin breakdown, eschar and ulcer were successfully produced sequentially by repeated compression in the rat abdominal wall, and the repair process was observed for a considerably long time. These injuries closely match human pressure ulcers (Bansal et al., 2005; Anders et al., 2010). The present experimental model is highly reproducible and needs neither anesthesia during compression nor special instruments except for gold-plated magnets. By this procedure, a gold-plated magnet can be implanted for months without any problems as long as the gold layer is intact. Alternatively, the magnet can be removed by operation after completing compressions at any time to avoid possible future problems. The wide abdominal wall has a roughly uniform structure consisting of several layers and is therefore appropriate for quantitative assessment. Changes in thickness of the abdominal wall could be evaluated by measuring the thickness of inserted acrylic plate(s) (Fig. 4). This method readily allows repeated compression and decompression. Thus, the magnitude of pressure, exposure time, and incidences of compressions are easily changeable to produce a variety of injuries similar to human pressure ulcers. This model provides relatively large specimens, up to $25 \times 20 \mathrm{~mm}$. In addition, a common small animal model, for example, the rat, offers the advantages of low initial cost and maintenance for an investigation (Peirce et al., 2000; Stekelenburg et al., 2005).

To produce pressure ulcers, the use of magnets has been reported in the literature (Peirce et al., 2000; Reid et al., 2004; Şener et al., 2006; Saito et al., 2008; Wassermann et al., 2009). However, the pressure in previous studies was either not measured or was not as accurate as in our experiment. The magnitude of pressure in the present investigation $(100 \mathrm{mmHg})$ is accurate and actually encountered in clinical situations. It is reported that the pressure on the sacrum when the subject is semirecumbent and with backrest at 45 degrees is 62-107 $\mathrm{mmHg}(8.3-14.3 \mathrm{kPa})$, and the pressure on the ischial tuberosities when sitting is $60-146 \mathrm{mmHg}(8.0-19.5$ $\mathrm{kPa}$ ) (Swain, 2005). In addition, the pressure of the abdominal wall sandwiched by two magnets is probably homogeneous while, in the case of an indenter, interface pressure is not so accurate (Linder-Ganz and Gefen, 2004; Oomens et al., 2010) owing to a sling or suspension effect (Kosiak, 1959).

In previous studies, a magnet or steel plate was implanted in the subcutaneous connective tissue (Peirce et al., 2000) or under the gluteus maximus muscle (Wassermann et al., 2009). In these cases, the healing process after skin breakdown is probably compromised by the implanted magnet or steel plate. However, the healing process in the present study was essentially unaffected by

Fig. 5. Fine structure of the rat abdominal wall. The figures at the upper left corner indicate the times of compression and the days after the last compression. D: dermis, S: skin muscle, M: abdominal muscle, E: eosinophilic fibrillar material. a: Normal untreated rat. Subcutaneous connective tissue (arrow) is thin. b: At 1 day after 2 compressions. The skin and subcutaneous connective tissue are significantly edematous and swelled. Hair follicles are sparse. Skin muscle (S) and abdominal muscle (M) are necrotic. Bundles of eosinophilic fibrillar material (E) are seen in the remarkably thickened subcutaneous connective tissue. Inset: An enlarged image of $\mathrm{E}$ in the boxed area. c: At 1 day after 4 compressions. Findings are similar to those at 1 day after 2 compressions. Sebaceous glands are decreased in number. d: At 2 days after 5 compressions. The border of the dermis is blurred due to loss of the skin muscle. e: At 4 days after 5 compressions. f: At 8 days after 5 compressions. g: At 12 days after 5 compressions. h: At 16 days after 5 compressions. i: At 20 days after 5 compressions. The epidermis is regenerated while the hair follicle, sebaceous gland and skin muscle are not restored. Bar=1 $\mathrm{mm}$ 
the implanted magnet in the peritoneal cavity. Although $4 \mathrm{~h}$ of exposure may be slightly long, this model is clinically more relevant than those in previous studies. The exposure time may be shortened, if preferable.

The abdominal wall apparently contracted a few days after 5 compressions, while injured tissue became very hard. A similar phenomenon was also observed in the greater trochanter of minipigs (Reger et al., 1999). Contraction of the wounds is probably more enhanced in the abdominal wall than in the greater trochanter or other parts of the body because the former is free from underlying tissues, which may disturb contraction. However, the mechanisms and relationship between contraction and hardening of the injured tissue remain unknown and call for further study.

Hair loss was occasionally noticed in the skin in mild and some moderate injuries, and poor regeneration of hair follicles and sebaceous glands was confirmed by light microscopy. On the other hand, hair loss was less frequently observed in severe injuries. These findings suggest that severely degenerated skin is almost completely removed by necrosis and repaired by extension of the surrounding normal skin. The discovery of normal-looking skin on the degenerated subcutaneous connective tissue supports this idea. Mild and moderately injured skin such as that with redness and erosion probably survives and is repaired by regeneration of the epidermis and injured tissues - although restoration of the normal structure of the skin and subcutaneous connective tissue takes a long time.

Concerning microscopic findings, marked edema and swelling were observed after 2 compressions but were almost unchanged thereafter. This result is in agreement with the change in thickness of acrylic plate(s), which reflects the swelling of the abdominal wall (Fig. 4). Light microscopic findings of the abdominal walls were essentially similar at 1 day after 2 or 4 compressions and at 2 days after 5 compressions. On the other hand, at the macroscopic level, the fates of these compressed abdominal skins significantly differ. On the basis of our preliminary experiments, it is assumed that the majority of the damage to the skin after 4 compressions can be reversed to full healing, whereas the injured skin after 5 compressions mainly proceeds to full-thickness necrosis and eschar formation. No critical difference could be distinguished between these specimens by light microscopy. In addition, the mechanism of hardening of the injured skin is unknown, and those factors that determine the fate of both injured skin and tissue hardening need to be clarified.

In summary, a clinically more relevant model of experimental pressure ulcers was successfully developed and the repair process was observed. This model should prove helpful for better understanding of the pathogenesis and progression of pressure ulcers and contribute to developing interventions for the prevention and treatment of pressure ulcers.

\section{References}

Anders J, Heinemann A, Leffmann C, Leutenegger M, Pröfener F, von Renteln-Kruse W: Decubitus ulcers: pathophysiology and primary prevention. Dtsch Arztebl Int 107: 371-81 (2010).

Bansal C, Scott R, Stewart D, Cockerell CJ: Decubitus ulcers: a review of the literature. Int $J$ Dermatol 44: 805-810 (2005).

Bosboom EM, Bouten CV, Oomens CW, van Straaten HW, Baaijens FP, Kuipers H: Quantification and localisation of damage in rat muscles after controlled loading; a new approach to study the aetiology of pressure sores. Med Eng Phys 23: 195-200 (2001).

Cordeiro MBC, Antonelli ÉJ, da Cunha DF, Júnior AAJ, Júnior VR, Vannucchi H: Oxidative stress and acute-phase response in patients with pressure sores. Nutrition 21: 901-907 (2005).

Daniel RK, Priest DL, Wheatley DC: Etiologic factors in pressure sores: an experimental model. Arch Phys Med Rehabil 62: 492-498 (1981).

Hashimoto M, Kurose T, Kawamata S: Comparison between a weight compression and a magnet compression for experimental pressure ulcers in the rat. histological studies and effects of anesthesia. Arch Histol Cytol 71: 303-316 (2008).

Kokate JY, Leland KJ, Held AM, Hansen GL, Kveen GL, Johnson BA, Wilke MS, Sparrow EM, Iaizzo PA. Temperature-modulated pressure ulcers: a porcine model. Arch Phys Med Rehabil 76: 666-673 (1995).

Kosiak M: Etiology and pathology of ischemic ulcers. Arch Phys Med Rehabil 40: 62-69 (1959).

Lin F, Pandya A, Cichowski A, Modi M, Reprogle B, Lee D, Kadono N, Makhsous M: Deep tissue injury rat model for pressure ulcer research on spinal cord injury. J Tissue Viability 19: 67-76 (2010).

Linder-Ganz E, Gefen A: Mechanical compressioninduced pressure sores in rat hindlimb: muscle stiffness, histology, and computational models. J Appl Physiol 96: 2034-2049 (2004).

Linder-Ganz E, Gefen A: The effects of pressure and shear on capillary closure in the microstructure of skeletal muscles. Ann Biomed Eng 35: 2095-2107 
(2007).

Oomens CWJ, Loerakker S, Bader DL: The importance of internal strain as opposed to interface pressure in the prevention of pressure related deep tissue injury. $J$ Tissue Viability 19: 35-42 (2010).

Peirce SM, Skalak TC, Rodeheaver GT: Ischemiareperfusion injury in chronic pressure ulcer formation: a skin model in the rat. Wound Rep Reg 8: 68-76 (2000).

Reger SI, Hyodo A, Negami S, Kambic HE, Sahgal V: Experimental wound healing with electrical stimulation. Artif Organs 23: 460-462 (1999).

Reid RR, Sull AC, Mogford JE, Roy N, Mustoe TA: A novel murine model of cyclical cutaneous ischemiareperfusion injury. J Surg Res 116: 172-180 (2004).

Saito Y, Hasegawa M, Fujimoto M, Matsushita T, Horikawa M, Takenaka M, Ogawa F, Sugama J, Steeber DA, Sato S, Takehara K: The loss of MCP-1 attenuates cutaneous ischemia-reperfusion injury in a mouse model of pressure ulcer. J Invest Dermatol 128: 1838-1851 (2008).
Şener G, Sert G, Özer Şehirli A, Arbak S, Uslu B, Gedik N, Ayanoglu-Dulger G: Pressure ulcer-induced oxidative organ injury is ameliorated by $\beta$-glucan treatment in rats. Int Immunopharmacol 6: 724-732 (2006).

Stekelenburg A, Oomens C, Bader D: Compressioninduced tissue damage: animal models. In : Pressure Ulcer Research. Current and Future Perspectives (Bader D, Bouten C, Colin D, Oomens C ed), SpringerVerlag, Heidelberg, 2005 (p.187-204).

Stekelenburg A, Oomens CW, Strijkers GJ, Nicolay K, Bader DL: Compression-induced deep tissue injury examined with magnetic resonance imaging and histology. J Appl Physiol 100: 1946-1954 (2006).

Swain Y: The measurement of interface pressure. In : Pressure ulcer research. Current and Future Perspectives (Bader D, Bouten C, Colin D, Oomens C ed), Springer-Verlag, Heidelberg, 2005 (p. 51-71).

Wassermann E, van Griensven M, Gstaltner K, Oehlinger W, Schrei K, Redl H: A chronic pressure ulcer model in the nude mouse. Wound Rep Reg 17: 480-484 (2009). 